\title{
NLPOTENT INJECTORS AND CONJUGACY CLASSES IN SOLVABLE GROUPS
}

\author{
GEOFFREY R. ROBINSON \\ To Laci Kovács on his 65 th birthday
}

(Received 11 October 2000)

Communicated by R. A. Bryce

\begin{abstract}
We provide an upper bound for the order of a nilpotent injector of a finite solvable group with Fitting subgroup of order $n$. We also show that the same bound is an upper bound for the number of conjugacy classes, provided that the $k(G V)$-conjecture holds for solvable $G$ all primes dividing $n$.
\end{abstract}

2000 Mathematics subject classification: primarỳ 20E45, $20 \mathrm{~F} 16$.

\section{Introduction}

There has been much progress lately towards the so-called $k(G V)$-problem, which asserts that if $G$ is a finite $p^{\prime}$-group and $V$ is a faithful $G F(p) G$-module, then $k(G V)$, the number of conjugacy classes of the semi-direct product $G V$, is at most $|V|$. In particular, by the results of [1] and [3] (which themselves built on a good deal of earlier work by several authors), if $G$ is solvable, then the $k(G V)$-problem is answered in the affirmative for all primes $p$ other than $3,5,7$ or 13 (the other cases still being open to date).

If the $k(G V)$-problem has an affirmative answer for the prime $p$ and solvable $G$, it follows more generally that we have $k(H) \leq|H|_{p}$ whenever $p$ is a prime and $H$ is a finite solvable group such that $F(H)$ is a $p$-group (as usual, for an integer $n$ and a prime $p$, we let $n_{p}$ denote the highest power of $p$ which divides $n$ ).

(C) 2001 Australian Mathematical Society $0263-6115 / 2001 \$ A 2.00+0.00$ 
This suggests the problem of finding other bounds for $k(G)$ in terms of distinguished subgroups of $G$, especially when $G$ is solvable. In a private communication to the author [5], Thompson asked whether it is the case that $k(G) \leq|I|$ whenever $G$ is solvable and $I$ is a nilpotent injector of $G$. There are several definitions of nilpotent injector in the literature, all of which coincide for solvable groups. For our purposes, we remind the reader that the nilpotent subgroup $I$ of the solvable group $G$ is a nilpotent injector if $I \cap N$ is a maximal nilpotent subgroup of $N$ whenever $N \triangleleft \triangleleft G$. Nilpotent injectors were first shown to exist for solvable groups by Fischer. They are unique up to conjugacy, and whenever $I$ is a nilpotent injector of a finite solvable group $G$, we have $O_{p}(I) \in \operatorname{Syl}_{p}\left(C_{G}\left(O_{p^{\prime}}(G)\right)\right)$ for each prime $p$.

In [4], Kovács and the author proved that if $G$ is solvable and $|F(G)|=p^{r}$ for some integer $r$, then $k(G) \leq 3^{r-1}|F(G)|$. It is not difficult to extend this result to prove that if $G$ is solvable and $|F(G)|$ has $\mathrm{r}$ prime factors (counting multiplicities), then $k(G) \leq 3^{r-1}|F(G)|$. We aim here to improve this bound somewhat.

We define the function $f: \mathbb{N} \rightarrow \mathbb{N}$ as follows: $f(1)=1 ; f(a b)=f(a) f(b)$ whenever $a$ and $b$ are relatively prime. When $p$ is an odd prime which is not Fermat, $f\left(p^{s}\right)=p^{s}(s !)_{p}$.

Whenever $p$ is a Fermat prime, and $s$ is an integer of the form $(p-1) t+u$ where $t$, $u$ are integers with $0 \leq u \leq p-2$, we set $f\left(p^{s}\right)=p^{s+t}(t !)_{p}$.

When $p=2$ and $s$ is a non-negative integer of the form $3 t+u$, where $t, u$ are integers with $0 \leq u \leq 2$, we set $f\left(2^{s}\right)=2^{s+3 t}(t !)_{2} u !$.

In this note, we prove the following:

THEOREM. Let $f$ be the function defined as above on the natural numbers. Then

(i) For each finite solvable group $H$, the order of a nilpotent injector $I$ of $H$ is a divisor of $f(|F(H)|)$. Furthermore, for each positive integer $n$ there exists a finite solvable group $H_{n}$ with Fitting subgroup of order $n$ and a nilpotent injector of order $f(n)$.

(ii) If $H$ is a solvable group such that the $k(G V)$-problem has an affirmative answer for solvable $G$ for all prime divisors of $|F(H)|$, then we have $k(H) \leq$ $f(|F(H)|)$.

Proof. It follows from a theorem of Winter [6] for odd primes, and the exposition and expansion of Winter's result in Isaacs' book [2], that whenever $p^{s}$ is a prime-power, $f\left(p^{s}\right) / p^{s}$ is an upper bound for the order of a Sylow $p$-subgroup of a completely reducible $p$-solvable subgroup of $G L(s, p)$ (actually, for $p=2$, the result in Isaacs' book gives the slightly weaker bound $2^{4 s-3 / 3}$, which agrees with $f\left(2^{s}\right) / 2^{s}$ when $s$ has the form $3 \cdot 2^{m}$ for some non-negative integer $m$. However, careful examination of the arguments gives the result in the above sharper form).

Furthermore, it is clear that each of these bounds may be realised in solvable 
subgroups of $G L(s, p)$. For example, if $p$ is odd, but not Fermat, then $C_{2}$ ? $P$ embeds as a completely reducible subgroup of $G L(s, p)$, where $P$ is a Sylow $p$-subgroup of the symmetric group of degree $s$. If $p=2$, then $X<P$ embeds as a completely reducible subgroup of $G L(s, 2)$, when $s$ has the form $3 t$ for some integer $t$, where $X$ is the semi-direct product of an extra-special group of order 27 with $S L(2,3)$ in its natural action, and $P$ is a Sylow 2-subgroup of the symmetric group of degree $t$ (and $(X, P) \times S_{3}$ embeds in $G L(s+2,2)$. If $p$ is a Fermat prime, then the semidirect product of an extra-special 2-group of order $2(p-1)^{2}$ with a cyclic group of order $p$ (with non-trivial action) embeds as an absolutely irreducible subgroup of $G L(p-1, p)$. Its wreath product with a Sylow $p$-subgroup of the symmetric group of degree $r$ embeds completely reducibly in $G L(r(p-1), p)$.

Taking an appropriate semi-direct product produces, for each prime $p$ and each non-negative integer $s$, a finite solvable group $H$ with $F(H)$ of order $p^{s}$ having a Sylow $p$-subgroup (which in this situation is a nilpotent injector) of order $f\left(p^{s}\right)$. Taking suitable direct products produces, for each positive integer $n$, a finite solvable group $H_{n}$ with $F\left(H_{n}\right)$ of order $n$ such that $H_{n}$ has a nilpotent injector of order $f(n)$.

On the other hand, we prove that a finite solvable group $H$ with $F(H)$ of order $n$ has a nilpotent injector of order dividing $f(n)$. It is useful for what follows to note the obvious fact that $f\left(p^{s}\right) / p^{s}$ increases with $s$. Consequently, as $F(H / \Phi(H))=$ $F(H) / \Phi(H)$, it suffices to assume that $\Phi(H)=1$, which we do. In that case, $F(H)$ is Abelian of squarefree exponent, and $H / F(H)$ acts completely reducibly on it.

Let $I$ be a nilpotent injector of $H$. Then $O_{p}(I) / O_{p}(H)$ acts faithfully on $O_{p}(H)$ as $O_{p}(I)$ acts trivially on $O_{p^{\prime}}(H)$. If $\left|O_{p}(H)\right|=p^{s}$, then $H / C_{H}\left(O_{p}(H)\right)$ acts completely reducibly on $O_{p}(H)$, so that $\left|O_{p}(I)\right| \leq f\left(p^{s}\right)$. Hence $I$ has order dividing $f(n)$, as $p$ was arbitrary.

It remains to prove that $k(H) \leq f(n)$ when $H$ is solvable with $|F(H)|=n$. Let $I$ be a nilpotent injector of $H$. We recall the well-known inequality $k(H) \leq k(N) k(H / N)$ whenever $N \triangleleft H$. This again allows us to assume that $\Phi(H)=1$, which we do. Set $F=F(H)$ and $N=O_{p}(F)$ for a prime $p$ dividing $|F|$. Then $F$ is completely reducible as $H / F$-module. Hence there is a subgroup $L$ of $H$ which complements $N$. It is routine to check that $F=N \times F\left(C_{L}(N)\right)$. Set $C=C_{L}(N) \triangleleft H$.

We have $O_{p}(C) \leq O_{p}(H)=N$, so that $F(C)=O_{p^{\prime}}(F(C))=O_{p^{\prime}}(F(H))$. Hence $O_{p^{\prime}}(I)=I \cap C$ is a nilpotent injector of $C$. We may assume by induction that $k(C) \leq f(|F(C)|)$. Now $H / C$ is the semi-direct product of $N$ with $L / C_{L}(N)$, with the latter group acting completely reducibly on $N$. Hence a Sylow $p$-subgroup of $H / C$ has order at most $f(|N|)$. Using the hypothesis that the $k(G V)$-problem has an affirmative answer for $p$, we see that $k(H / C) \leq f(|N|)$. Hence we see that $k(H) \leq k(C) k(H / C) \leq f(|F(C)|) f(|N|)=f(|F(H)|)$, as required to complete the proof. 


\section{References}

[1] D. Gluck and K. Magaard, 'The extra-special case of the $k(G V)$-problem', preprint, 1999.

[2] I. M. Isaacs, Character theory of finite groups, Pure Appl. Math. 69 (Academic Press, New York, 1976).

[3] C. Köhler and H. Pahlings, 'Regular orbits and the $k(G V)$-problem', in: Groups and computation III. (Columbus, OH, 1999) Ohio State Univ. Math. Res. Inst. Publ. 8 (deGruyter, Berlin, 2001),pp. 209-228.

[4] L. G. Kóvacs and G. R. Robinson, 'On the number of conjugacy classes of finite groups', J. Algebra 160 (1993), $441-460$.

[5] J. G. Thompson, private communication, (circa 1989).

[6] D. L. Winter, ' $p$-solvable linear groups of finite order', Trans. Amer. Math. Soc. 157 (1971), 155-160.

School of Mathematics and Statistics

University of Birmingham

Edgbaston

Birmingham B15 2TT

England 